\title{
RESEARCH ON THE DISTURBANCE GENERATED BY A SOLAR ARRAY DRIVE ASSEMBLY DRIVING A FLEXIBLE SYSTEM
}

\author{
JiangPan Chen, Wei Cheng \\ School of Aeronautic Science and Engineering, Beijing University of Aeronautics and Astronautics, Beijing, China \\ e-mail: chenjiangpan@hotmail.com; cheng_wei@buaa.edu.cn
}

\begin{abstract}
The present work is aimed at presenting the disturbance generated by a solar array drive assembly (SADA) driving a flexible system. Firstly, the vibration equation of SADA is obtained by simplifying and linearizing the electromagnetic torque. Secondly, the disturbance model of SADA driving a discrete flexible system is achieved based on the vibration equation established. Taking a two-dof flexible system as the study object, this disturbance model is simulated and analyzed. Lastly, a continuous flexible system, which is designed to simulate the solar array, is used to illustrate the simulation method of the disturbance emitted by SADA driving a continuous flexible system. All the achievements obtained from this project will provide a theoretical basis for the prediction of the disturbance emitted by the SADA driving solar array on the orbit.
\end{abstract}

Keywords: solar array drive assembly, disturbance, flexible system, electromagnetic torque, vibration equation

\section{Introduction}

Micro-vibrations of a satellite, which are characterized by a low amplitude and a wide frequency band, can significantly degrade its pointing accuracy and imaging quality. There are many possible disturbance sources, such as reaction/momentum wheel assembly, control moment gyroscope and SADA (Luo et al., 2013). Due to a longer exposure time than in a low orbit remote sensing satellite, the performance of a high orbit remote sensing satellite is more sensitive to micro-vibrations in the low frequency band. Three reasons make the disturbance emitted by the SADA driving solar array on the orbit extremely complex and mainly in the low frequency band, which are: (1) distribution of the natural frequency of the solar array in the low frequency band is dense (Cui, 2006); (2) exciting energy of SADA in the low frequency band is large, which makes the natural vibration of the solar array easily excited out; (3) vibration attenuation of the solar array in the low frequency band is slow without air damping in space. Therefore, to study the disturbance produced by the SADA driving solar array is imperative. Because of the gravity, the solar array, which has characteristics of low stiffness, large mass and large size (Chen, 2010), is difficult to unfold on the Earth. Testing of the disturbance generated by the SADA driving solar array in the atmospheric environment is hard to carry out. A disturbance model used to predict the disturbance emitted by the SADA driving solar array on the orbit is necessary.

From researches carried out thus far, lots of studies have focused on the disturbance properties of SADA. The mechanism of electromagnetic vibration of SADA was studied by Xia (1994), and the methods to lower the amplitude of the electromagnetic vibration were also proposed, such as using damping (including mechanical damping and electrical damping) and choosing a more appropriate drive circuit. The expression for electromagnetic vibration frequency of SADA was presented in Bodson et al. (2006). In Yang et al. (2010) and Zhu et al. (2015), argued that the electromagnetic field between the stator and rotor of SADA could be equivalent to an 
electromagnetic spring-viscous damping system. Stiffness of the electromagnetic spring, named as electromagnetic stiffness, was obtained by experimental testing by Yang et al. (2010). Zhu et al. (2015) validated feasibility of the equivalent electromagnetic field to an electromagnetic spring-viscous damping system, and also investigated the coupling effect between the solar array and the electromagnetic spring-viscous damping system. Meanwhile, Si et al. (2010), Elsodany et al. (2011) and Szolc et al. (2012) proposed dynamic models of SADA. Among them, the dynamic model proposed by Si et al. (2010) was more complete, in which the friction torque and fluctuation torque of SADA were taken into account. The disturbance emitted by SADA driving a rigid system could be obtained by the dynamic models easily. Chen et al. (2014) modeled and simulated the disturbance produced by SADA driving a rigid system through a flexible transmission shaft based on the dynamic model of SADA. In this paper, the coupling effect between the electromagnetic stiffness and the flexible transmission shaft was studied by changing the stiffness of the transmission shaft.

However, the electromagnetic stiffness of the electromagnetic spring has not appeared in the dynamic models as an explicit expression up to now, and just has been hidden in the electromagnetic torque, which prevented the establishment of the coupling effect between the electromagnetic stiffness and the flexible system. As the solar array is an extremely complex typical continuous flexible system, the dynamic models existing do not have the ability to describe the disturbance caused by the SADA driving solar array on the orbit precisely and easily.

As a consequence, in this paper, the first part is to establish the vibration equation of SADA; the second part is to model, simulate and analyze the disturbance produced by SADA driving a discrete flexible system; the third part is to introduce the simulation method of the disturbance generated by SADA driving a continuous flexible system; the last part summarizes the paper and states conclusions drawn from this work.

\section{Modeling of the vibration equation of SADA}

The SADA discussed in this paper is a two-phase hybrid stepper motor. Stepper motors are motors which translate digital pulse sequences to precise movements of the rotor shaft, rather than rotating continuously as conventional motors do (Anish et al., 2012). Because of the advantages of high resolution, high torque and low noise, the stepper motor is widely used in the field of spacecraft (Letchmanan et al., 2005). Both solar array and digital antenna use stepper motors as the driver. The most frequently used dynamic model of SADA at present is given by

$$
J_{0} \ddot{\theta}=T_{e}-C_{0} \dot{\theta}-T_{l}
$$

where $J_{0}$ is the moment of inertia of the rotor, $\theta$ is the angular displacement of the rotor, $T_{e}$ is the electromagnetic torque, $C_{0}$ is the viscous damping coefficient and $T_{l}$ is the load torque.

As to a two-phase hybrid stepper motor, when the linear flux model without saturation effects is assumed, the driving model presented by Kapun et al. (2007) can be used in the form

$$
\boldsymbol{\varphi}=\mathbf{L I} \quad T_{e}=\frac{1}{2} \mathbf{I}^{\mathrm{T}} \frac{\partial \boldsymbol{\varphi}}{\partial \theta}=\frac{1}{2} \mathbf{I}^{\mathrm{T}} \frac{\partial \mathbf{L}}{\partial \theta} \mathbf{I}
$$

where $\varphi$ is the flux linkage vector, $\mathbf{L}$ is the symmetric inductance matrix and $\mathbf{I}$ is the current vector. The expressions of $\mathbf{I}$ and $\mathbf{L}$ are given by

$$
\mathbf{I}=\left[\begin{array}{c}
I_{A} \\
I_{B} \\
I_{f}
\end{array}\right] \quad \mathbf{L}=\left[\begin{array}{ccc}
L_{A A} & L_{A B} & L_{A f} \\
L_{B A} & L_{B B} & L_{B f} \\
L_{f A} & L_{f B} & L_{f f}
\end{array}\right]
$$


where $I_{A}$ and $I_{B}$ signifies the current of phase $A$ and phase $B$ respectively, $I_{f}$ is the fictitious constant rotor current, $L_{i i}(i=A, B)$ is the self-inductance of the stator winding, $L_{A B}=L_{B A}$ is the mutual inductance between the stator windings, $L_{f f}$ is the self-inductance of the fictitious rotor winding, $L_{i f}=L_{f i}(i=A, B)$ is the mutual inductance between the $i$-th stator winding and the fictitious rotor winding, see Yang et al. (2010) and Kapun et al. (2007).

Assuming that the mutual inductance between the stator windings is negligible and ignoring the second and more harmonics of the cyclical permeance function, the elements of the inductance matrix $\mathbf{L}$ can be defined as

$$
\begin{aligned}
& L_{A A}=L_{B B}=L_{0} \quad L_{A B}=L_{B A}=0 \quad L_{A f}=L_{f A}=L_{m 0}+L_{m 1} \cos (z \theta) \\
& L_{B f}=L_{f B}=L_{m 0}+L_{m 1} \sin (z \theta) \quad L_{f f}=L_{f 0}+L_{f 1} \cos (4 z \theta)
\end{aligned}
$$

where $z$ is the rotor teeth number. Substituting equations (2.3) and (2.4) into equation (2.2), the electromagnetic torque can be obtained as

$$
T_{e}=T_{m}-T_{d}=K_{m}\left[I_{B} \cos (z \theta)-I_{A} \sin (z \theta)\right]-K_{d} \sin (4 z \theta)
$$

where $T_{m}$ is the motor torque, $T_{d}$ is the detent torque, $K_{m}=z I_{f} L_{m 1}$ and $K_{d}=2 z I_{f}^{2} L_{f 1}$ is the motor torque constant and detent torque constant, respectively (Letchmanan et al., 2005).

In general, the detent torque constant $K_{d}$ is so small that the detent torque $T_{d}$ is far less than the motor torque $T_{m}$, and can be ignored (Yang et al., 2010). Thus, equation (2.5) can be simplified as

$$
T_{e}=K_{m}\left[I_{B} \cos (z \theta)-I_{A} \sin (z \theta)\right]
$$

In order to improve the resolution and running smoothness of the stepper motor, a subdivision driver is frequently used. Yang et al. (2007) pointed out that the ladder sine curve current is frequently used as the subdivision driving current in the subdivision driver. Thus, the expression for the two-phase current $I_{A}$ and $I_{B}$ after subdivision can be shown as

$$
I_{A}=I \cos (\gamma i) \quad I_{B}=I \sin (\gamma i)
$$

where $I$ is the amplitude of the two-phase current, $\gamma$ is the electrical step angle after subdivision and $i=1,2, \ldots$ is the step number. Substituting equation (2.7) into equation (2.6), the electromagnetic torque can be rewritten as

$$
T_{e}=K_{m} I \sin (\gamma i-z \theta)=K_{m} I \sin \left[z\left(\frac{\gamma i}{z}-\theta\right)\right]=K_{m} I \sin (z \Delta \theta)
$$

In equation (2.8), the physical meaning of $\Delta \theta$ is that: $\Delta \theta$ is the included angle between the actual position and theoretical equilibrium position of the rotor during the time period that after the input of the $i$-th digital pulse signal completed and before the input of $(i+1)$-th digital pulse signal, where $\gamma i / z$ and $\theta$ is the theoretical equilibrium position and actual position of the rotor in this time period, respectively. To ensure SADA running without losing step, the range of $\Delta \theta$ is given by

$$
-\alpha \leqslant \Delta \theta \leqslant \alpha
$$

where $\alpha$ is the micro-step angle after subdivision. The expression for $\alpha$ is given by

$$
\alpha=\frac{2 \pi}{z p n}
$$

where $p$ is the beat number and $n$ is the subdivision number. The value of $p$ and $n$ of the SADA studied in this paper is 4 and 256 , respectively, thus, we can obtain that

$$
-\frac{\pi}{512} \leqslant z \Delta \theta \leqslant \frac{\pi}{512}
$$


A significant result can be obtained from equation (2.11) that: $z \Delta \theta$ is so small that equation (2.8) can be linearized as

$$
T_{e}=K_{m} I \sin (z \Delta \theta)=K_{m} I z \Delta \theta=K_{m} I \gamma i-K_{m} I z \theta
$$

Substituting equation (2.12) into equation (2.1), the dynamic model of SADA can be rewritten as

$$
J_{0} \ddot{\theta}+C_{0} \dot{\theta}+K_{0} \theta=K_{m} I \gamma i-T_{l}
$$

where $K_{0}=K_{m} I z$ and $K_{m} I \gamma i$ is called the electromagnetic stiffness and exciting torque, respectively.

From equation $(2.13)$ we can get that: 1) the electromagnetic field between the stator and rotor of SADA can be equivalent to an electromagnetic spring-viscous damping system; 2) the rotor of SADA coupled with the load vibrates with viscous damping under the action of the exciting torque $K_{m} I \gamma i$. Thus, the equivalent system diagram of SADA can be shown as Fig. 1 .

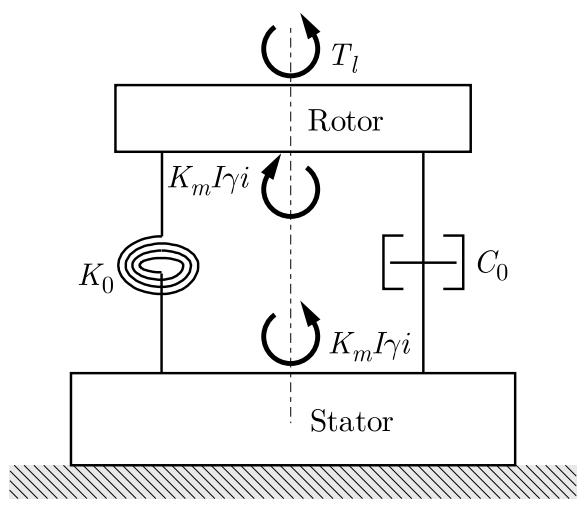

Fig. 1. Equivalent system of SADA

Assuming that the stator of SADA is a rigid body, the disturbance torque generated by SADA acting on the satellite can be defined as

$$
T_{d i s}=K_{m} I \gamma i-C_{0} \dot{\theta}-K_{0} \theta
$$

Thus, the disturbance model of SADA can be defined as

$$
J_{0} \ddot{\theta}+C_{0} \dot{\theta}+K_{0} \theta=K_{m} I \gamma i-T_{l} \quad T_{d i s}=K_{m} I \gamma i-C_{0} \dot{\theta}-K_{0} \theta
$$

From equation (2.15) we can obtain that the dynamic model of SADA can be redefined as a vibration equation, and the electromagnetic stiffness appears in this vibration equation as an explicit expression which leads to the coupling relationship between the electromagnetic spring-viscous damping system and the solar array easily achieved by establishing the vibration equation of the coupling system. Thus, the disturbance torque aroused by the SADA driving solar array acting on the satellite can be facilely obtained.

\section{Disturbance model of SADA driving a discrete flexible system}

\section{1. $\quad$ Modeling}

Assuming that the discrete flexible system is a torsion spring-damping-moment of inertia system with $n$-dofs, the equivalent system of SADA driving this discrete flexible system is shown in Fig. 2. 


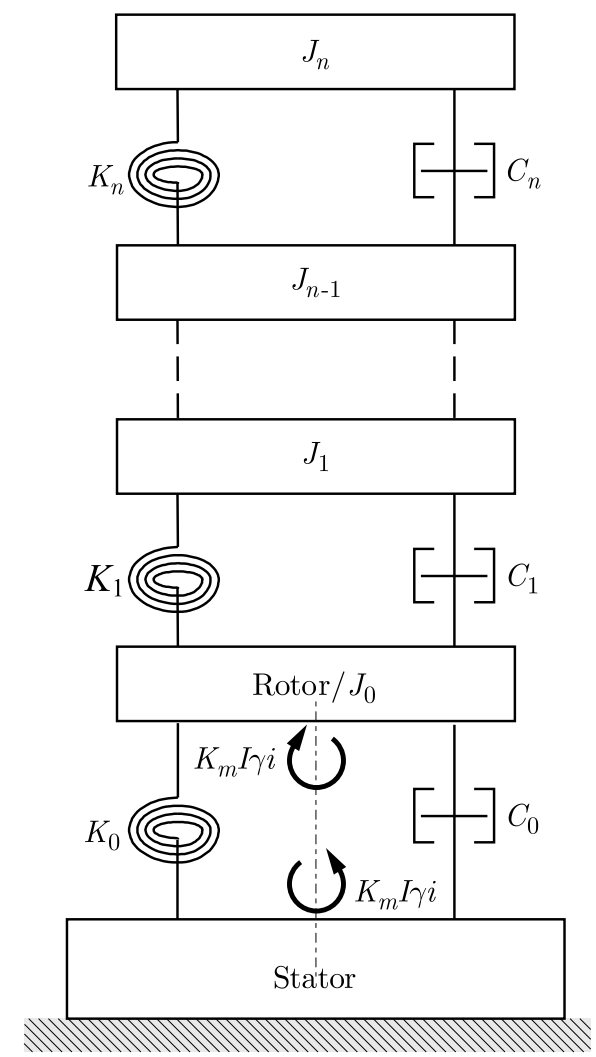

Fig. 2. Equivalent system diagram of SADA driving a discrete flexible system with $n$-dofs

Thus, the disturbance model of SADA driving this flexible system can be defined as

$$
\mathbf{J} \ddot{\boldsymbol{\beta}}+\mathbf{C} \dot{\boldsymbol{\beta}}+\mathbf{K} \boldsymbol{\beta}=\mathbf{F} \quad T_{d i s}=K_{m} I \gamma i-C_{0} \dot{\theta}-K_{0} \theta
$$

where $\mathbf{J}, \mathbf{C}, \mathbf{K}, \mathbf{F}$ and $\boldsymbol{\beta}$ is the mass matrix, damping matrix, stiffness matrix, excitation vector and angular displacement vector, respectively. All the expressions are shown in equation (3.2)

$$
\begin{aligned}
& \mathbf{J}=\left[\begin{array}{cccc}
J_{0} & 0 & \cdots & 0 \\
0 & J_{1} & \cdots & 0 \\
\vdots & \vdots & \ddots & \vdots \\
0 & 0 & \cdots & J_{n}
\end{array}\right] \quad \mathbf{C}=\left[\begin{array}{cccc}
C_{0}+C_{1} & -C_{1} & \cdots & 0 \\
-C_{1} & C_{1}+C_{2} & \cdots & 0 \\
\vdots & \vdots & \ddots & \vdots \\
0 & 0 & \cdots & C_{n}
\end{array}\right] \\
& \mathbf{K}=\left[\begin{array}{cccc}
K_{0}+K_{1} & -K_{1} & \cdots & 0 \\
-K_{1} & K_{1}+K_{2} & \cdots & 0 \\
\vdots & \vdots & \ddots & \vdots \\
0 & 0 & \cdots & K_{n}
\end{array}\right] \quad \mathbf{F}=\left[\begin{array}{c}
K_{m} I \gamma i \\
0 \\
\vdots \\
0
\end{array}\right] \quad \boldsymbol{\beta}=\left[\begin{array}{c}
\theta \\
\theta_{1} \\
\vdots \\
\theta_{n}
\end{array}\right]
\end{aligned}
$$

\subsection{Simulation and analysis}

Taking a two-dof torsion spring-damping-moment of inertia system as the simulation object, Matlab/Simulink toolbox is used to simulate the disturbance model of SADA driving this discrete flexible system. Table 1 shows the simulation parameters of SADA and the two-dof flexible system. The simulation time and time step size is $100 \mathrm{~s}$ and $1 / 2048 \mathrm{~s}$, respectively.

Figures 3 to 5 illustrate the simulation results of the disturbance model of SADA driving this two-dof flexible system.

It can be seen from Figs. 3a and 3b that the two-phase currents $I_{A}$ and $I_{B}$ are ladder cosine/sine curves, and the amplitude $I$ is $0.3 \mathrm{~A}$. 
Table 1. Simulation parameters of SADA and the two-dof flexible system

\begin{tabular}{|l|c|c|c|}
\hline Parameter & Symbol & Value & Unit \\
\hline \hline Rotor teeth number & $z$ & 300 & - \\
Beat number & $p$ & 4 & - \\
Subdivision number & $n$ & 256 & - \\
Given angular velocity of rotor & $\omega_{0}$ & 0.012 & $\circ / \mathrm{s}$ \\
Amplitude of two-phase current & $I$ & 0.3 & $\mathrm{~A}$ \\
Moment of inertia of rotor & $J_{0}$ & 0.0005 & $\mathrm{~kg} \cdot \mathrm{m}^{2}$ \\
Viscous damping coefficient & $C_{0}$ & 0.01 & $\mathrm{~N} \cdot \mathrm{m} \cdot \mathrm{s} / \mathrm{rad}$ \\
Motor torque constant & $K_{m}$ & 10 & $\mathrm{~N} \cdot \mathrm{m} / \mathrm{A} \cdot \mathrm{rad})$ \\
Electromagnetic stiffness & $K_{0}$ & 900 & $\mathrm{~N} \cdot \mathrm{m} / \mathrm{rad}$ \\
Moment of inertia of flexible system & $J_{1} / J_{2}$ & $1 / 4$ & $\mathrm{~kg} \cdot \mathrm{m}^{2}$ \\
Viscous damping coefficient & $C_{1} / C_{2}$ & $0.02 / 0.01$ & $\mathrm{~N} \cdot \mathrm{m} \cdot \mathrm{s} / \mathrm{rad}$ \\
Stiffness of torsion spring & $K_{1} / K_{2}$ & $1000 / 2000$ & $\mathrm{~N} \cdot \mathrm{m} / \mathrm{rad}$ \\
\hline
\end{tabular}
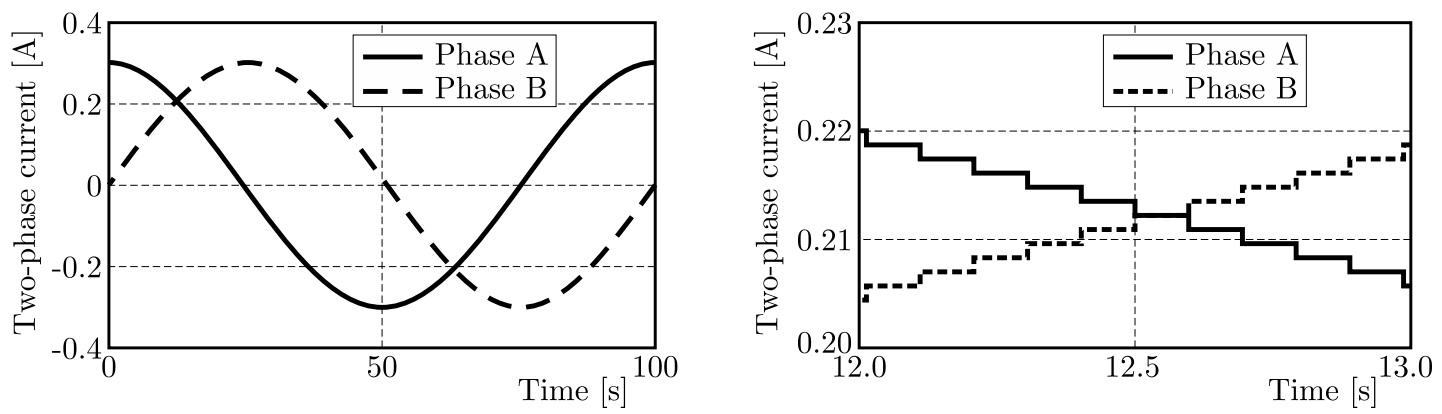

Fig. 3. Simulation results of the two-phase current (a) full view (b) partially enlarged view

As shown in Fig. 4, the simulation result of the rotor angular displacement indicates that after 100 seconds of running, the angular displacement of the rotor is $1.2^{\circ}$, which is equal to the given angular velocity $\omega_{0}\left(0.012^{\circ} / \mathrm{s}\right)$ times the running time $(100 \mathrm{~s})$.

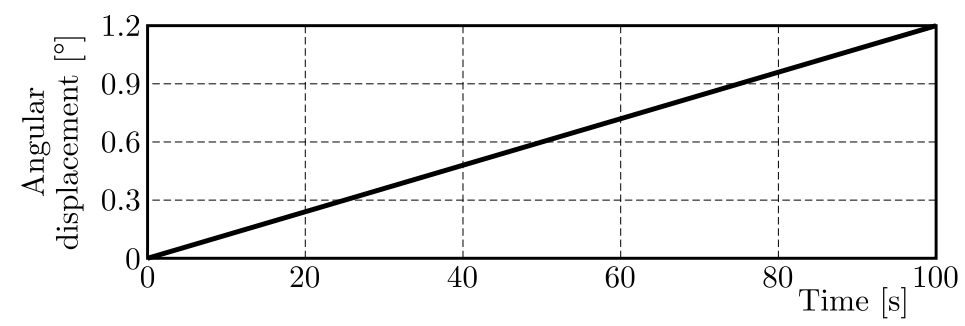

Fig. 4. Simulation result of the angular displacement of the rotor

The simulation result of the disturbance torque shown in Fig. 5 indicates that the disturbance frequencies in the frequency band of $0 \sim 40 \mathrm{~Hz}$ are $1.438 \mathrm{~Hz}, 8.563 \mathrm{~Hz}, 10.25 \mathrm{~Hz}, 20.5 \mathrm{~Hz}$ and $30.75 \mathrm{~Hz}$. Substituting the parameters shown in Table 1 into equation (3.2), the natural frequency of the equivalent system of SADA driving the two-dof flexible system can be calculated easily, which is demonstrated as

$$
f_{1}=1.440 \mathrm{~Hz} \quad f_{2}=8.558 \mathrm{~Hz} \quad f_{3}=310.272 \mathrm{~Hz}
$$

Therefore, the disturbance frequencies $1.438 \mathrm{~Hz}$ and $8.563 \mathrm{~Hz}$ are the natural frequencies of the equivalent coupling system of SADA driving the flexible system. 


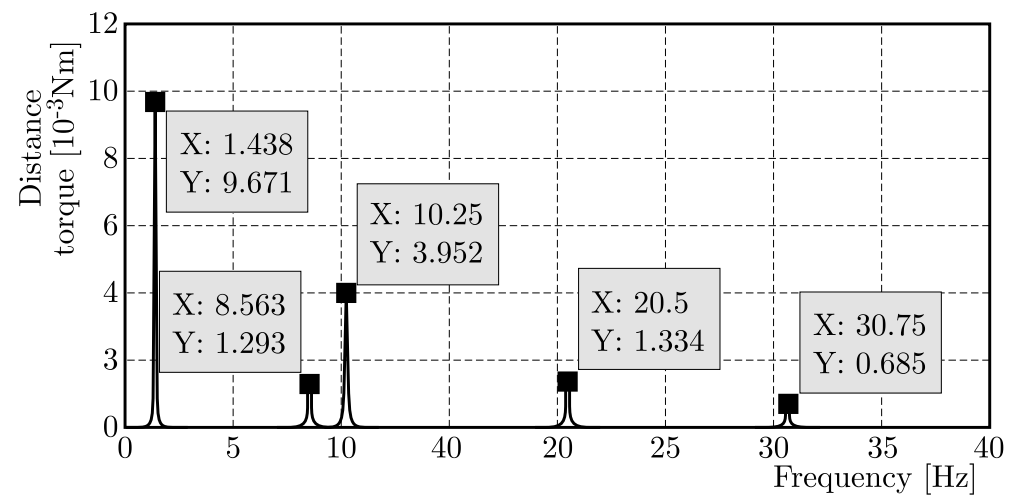

Fig. 5. Simulation result of the disturbance torque

As mentioned above, SADA is to translate digital pulse sequences to precise movements of the rotor shaft. The input frequency of the digital pulse signal is given by

$$
f_{d}=\frac{\frac{\pi \omega_{0}}{180}}{\alpha}=\frac{\frac{\pi \omega_{0}}{180}}{\frac{2 \pi}{z p n}}=10.24 \mathrm{~Hz}
$$

Thus, the disturbance frequencies $10.25 \mathrm{~Hz}, 20.5 \mathrm{~Hz}$ and $30.75 \mathrm{~Hz}$ are the input frequency of the digital pulse signal and its harmonics.

As a consequence, we can obtain that the disturbance frequencies of SADA driving a discrete flexible system are mainly consists of two parts: 1) natural frequencies of the equivalent system of SADA driving the flexible system; 2) input frequency of the digital pulse signal and its harmonics. These disturbance frequencies are consistent with those obtained by Yang et al. (2010) and Chen et al. (2014), which confirmedly validates the effectiveness of the disturbance model proposed in this paper.

\section{Simulation method of SADA driving a continuous flexible system}

Since the solar array is a typical continuous flexible system, equation (3.1) used to describe vibration of the equivalent system of the SADA driving solar array is difficult to establish. Taking a continuous flexible system, which is designed to simulate the solar array, as the studied object, the simulation method of the disturbance generated by SADA driving a continuous flexible system is demonstrated in this part.

\subsection{Continuous flexible system}

The continuous flexible system is made of a thin aluminum plate, steel counterweight beam and connecting bracket. The size of the thin aluminum plate is $5 \mathrm{~m} \times 1.6 \mathrm{~m} \times 0.001 \mathrm{~m}$. The steel counterweight beam is arranged along the four sides of the thin aluminum plate continuously, whose section size is $0.03 \mathrm{~m} \times 0.03 \mathrm{~m}$. The connection bracket is composed of two identical steel beams. The length of each steel beam is $1 \mathrm{~m}$, and its section size is $0.03 \mathrm{~m} \times 0.03 \mathrm{~m}$ as well. A schematic diagram of this continuous flexible system is shown in Fig. 6.

ANSYS 12.1 is used to create the finite element model of this continuous flexible system. In the finite element model, element SHELL 181 is used to mesh the thin aluminum plate and element BEAM 4 is used to mesh the steel counterweight beam and the connecting bracket. What is more, a node should be laid in the installation point which is named as node $a$. The modal shape of this finite element model is determined for the boundary condition of constraining all-dofs of node $a$. The calculation results of the modal analysis are listed in Table 2. 


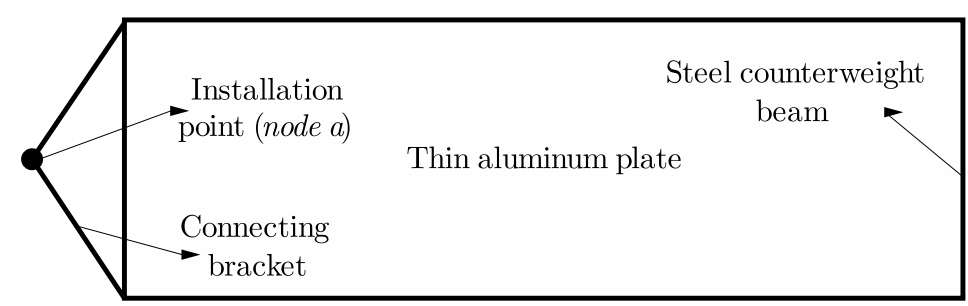

Fig. 6. Schematic diagram of the continuous flexible system

Table 2. Calculation results of the modal

\begin{tabular}{|c|c|c|}
\hline Modal order & Frequency $[\mathrm{Hz}]$ & Modal shape \\
\hline \hline 1 & 0.503 & first-order out-of-plane bending \\
2 & 1.364 & first-order in-plane rocking \\
3 & 2.110 & first-order torsional \\
4 & 2.222 & second-order out-of-plane bending \\
\hline
\end{tabular}

As the former four order modal shapes of the continuous flexible system designed are the same as the actual solar array mentioned in Zhu et al. (2014), this continuous flexible system has the ability to simulate the actual solar array.

\subsection{Continuous flexible system coupling with SADA}

As discussed above, the electromagnetic field between the stator and rotor of SADA can be equivalent to an electromagnetic spring-viscous damping system, thus, the equivalent system of the continuous flexible system coupling with SADA can be shown as in Fig. 7.

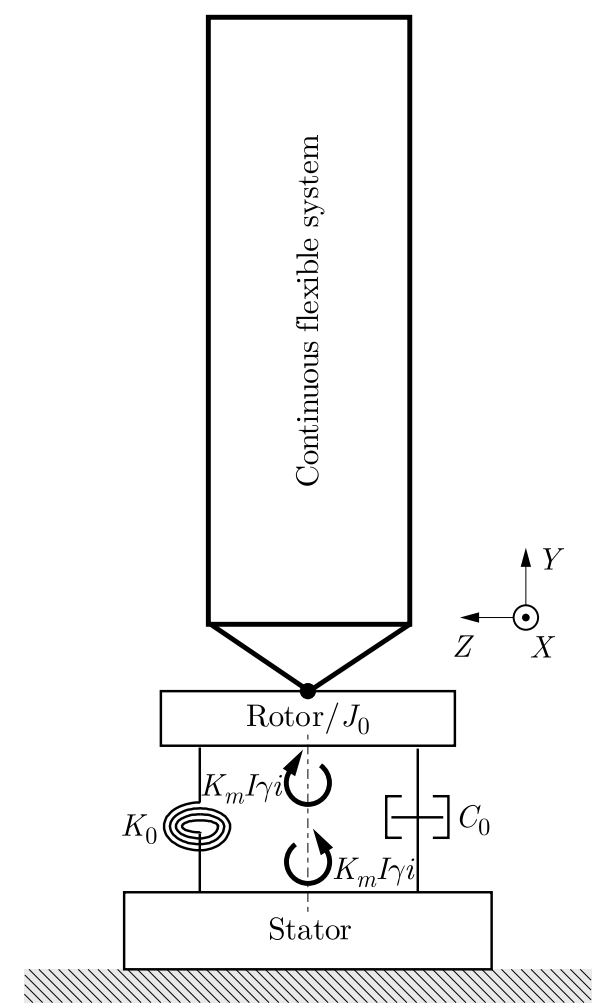

Fig. 7. Equivalent system of the continuous flexible system coupling with SADA 
ANSYS 12.1 is used to create the finite element model of this equivalent system. In this finite element model, the moment of inertia of the rotor $J_{0}$ is meshed by using element MASS 21; the electromagnetic torsion spring-viscous damping system is meshed by using element COMBIN 14; the finite element model of the continuous flexible system in the equivalent system is the same as the finite element model created above. Element MASS 21, element COMBIN 14 and the finite element model of the continuous flexible system should share one node, which is node $a$. As the rotor of SADA has only one rotation dof, node a has only this dof ( $Y$-rotation) as well. Constraining all the other dofs of node $a$ and all-dofs of the other node of element COMBIN 14, then the creation process of the finite element model is accomplished.

ANSYS 12.1 is used to find the modal shapes of this coupling finite element model. The calculation results of the modal analysis are presented in Table 3.

Table 3. Calculation results of the modal analysis

\begin{tabular}{|c|c|c|}
\hline Modal order & Frequency $[\mathrm{Hz}]$ & Modal shape \\
\hline \hline 1 & 0.503 & first-order out-of-plane bending \\
2 & 0.606 & first-order torsional \\
3 & 1.364 & first-order in-plane bending \\
4 & 2.222 & second-order out-of-plane bending \\
\hline
\end{tabular}

Comparing with the calculation results shown in Table 2, the natural frequency of the firstorder torsional modes moves forward and the other natural frequency remains the same, which is caused by the coupling effect between the continuous flexible system and the electromagnetic spring of SADA.

\subsection{Simulation and analysis}

ANSYS 12.1/Transient is used to simulate the disturbance emitted by SADA driving the continuous flexible system designed. The simulation process is done on the basis of the coupling finite element model created in Section 4.2. In the simulation processing, firstly, the exciting torque $K_{m} I \gamma i$ acts on node a; secondly, the simulation time and time step size is $20 \mathrm{~s}$ and $1 / 2048 \mathrm{~s}$ respectively; thirdly, each substep of the angular displacement and angular velocity of node $a$ are written down; lastly, substituting the angular displacement and angular velocity of node a into equation (3.1), the disturbance torque of SADA driving this continuous flexible system is obtained. The simulation results are shown in Figs. 8 and 9.

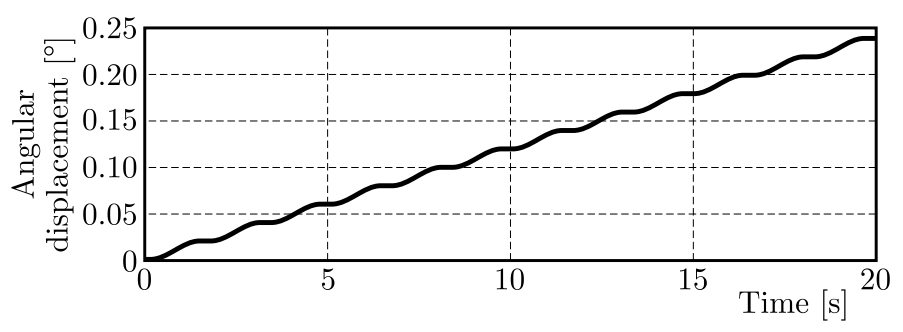

Fig. 8. Simulation result of the angular displacement of the rotor (node a)

It can be seen from Fig. 8 that due to huge torsional vibration of the continuous flexible system coupling with SADA in the low frequency band $(0.606 \mathrm{~Hz})$, the angular displacement of the rotor is no longer a straight line. However, after 20 seconds of running, the angular displacement of rotor is very close to $0.24^{\circ}$, which equals to the given angular velocity of the rotor $\omega_{0}\left(0.012^{\circ} / \mathrm{s}\right)$ times the running time $(20 \mathrm{~s})$.

What can be obtained from Fig. 9 are the disturbance frequencies in the frequency band of $0 \sim 40 \mathrm{~Hz}$ are $0.625 \mathrm{~Hz}, 10.25 \mathrm{~Hz}, 20.5 \mathrm{~Hz}$ and $30.75 \mathrm{~Hz}$. Among them, $0.625 \mathrm{~Hz}$ is the natural 


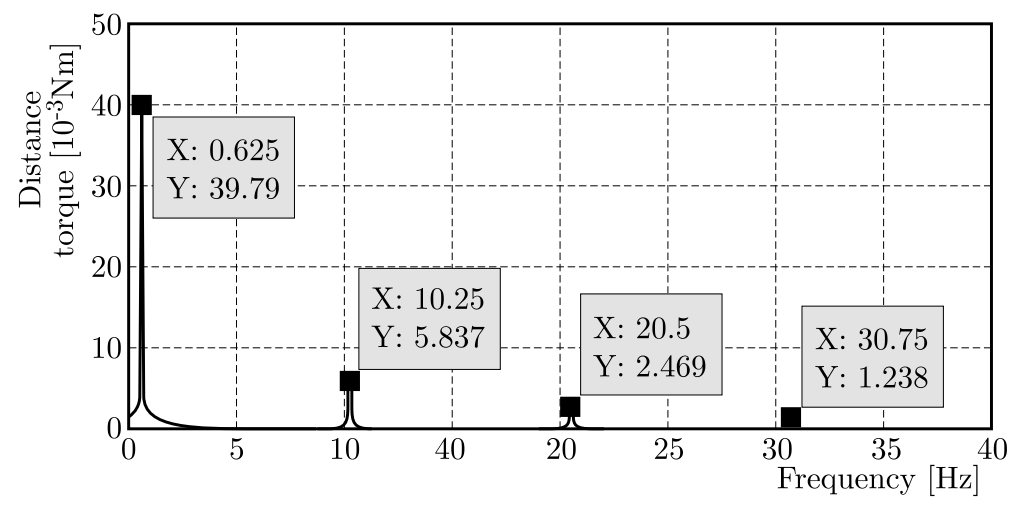

Fig. 9. Simulation result of the disturbance torque

frequency of the first-order torsional mode of the continuous flexible system coupled with SADA shown in Table $3 ; 10.25 \mathrm{~Hz}, 20.5 \mathrm{~Hz}$ and $30.75 \mathrm{~Hz}$ is the input frequency of the digital pulse signal and its harmonics. Due to low exciting energy of the exciting torque $K_{m} I \gamma i$ beyond the low frequency band and the existence of the viscous damping inner SADA, the natural frequencies of high-order torsional modes of the continuous flexible system coupled with SADA do not appear in the disturbance frequencies.

Therefore, the disturbance frequencies of SADA driving the continuous flexible system mainly consist of two parts: 1) natural frequencies of the torsional mode of the continuous flexible system coupled with SADA in the low frequency band; 2) input frequency of the digital pulse signal and its harmonics. These conclusions are the same as those obtained for SADA driving athe discrete flexible system in Section 3.2. This proves that the simulation method of SADA driving the continuous flexible system demonstrated in this part is right.

\subsection{Summarizing}

The simulation method of the disturbance aroused by SADA driving the continuous flexible system can be summarized as follows:

- Before the start of simulation, the finite element model of the continuous flexible system coupled with SADA, which can be equivalent to an electromagnetic spring-viscous damping system, should be created firstly, and the coupling node named node a. Appropriate boundary conditions should be formulated as well.

- In the process of simulation, the exciting torque $K_{m} I \gamma i$ should act on node $a$, and each substep of the angular displacement and angular velocity of node a should be written down. What is more, an appropriate simulation time and time step size should be chosen.

- After the end of simulation, substituting the angular displacement and angular velocity of node a into equation (3.1), the disturbance torque produced by SADA driving the continuous flexible system could be obtained.

\section{Conclusions}

According to the above discussions, the following conclusions can be drawn:

- By simplifying and linearizing the electromagnetic torque of SADA, the electromagnetic field between the stator and rotor of SADA can be found as equivalent to an electromagnetic spring-viscous damping system, and the vibration equation of SADA can be established. 
- The disturbance model of SADA driving a discrete flexible system is achieved based on the vibration equation of SADA. A two-dof flexible system is used to simulate and analyze the disturbance model. The simulation results show that the disturbance frequencies of SADA mainly consist of two parts:

1) natural frequencies of the equivalent system of SADA driving the flexible system;

2) input frequency of the digital pulse signal and its harmonics.

- The method to simulate the disturbance emitted by SADA driving the continuous flexible system, which is designed to simulate the solar array, is proposed. And the disturbance frequencies of SADA mainly consist of two parts:

1) natural frequencies of the low-order torsional mode of the continuous flexible system coupling with SADA in the low frequency band;

2) input frequency of the digital pulse signal and its harmonics.

In conclusion, all the achievements of this project will provide a theoretical basis for the prediction of disturbances emitted by the SADA driving solar array on the orbit.

\section{References}

1. Anish N.K., Krishnan D., Moorthi S., Selvan M.P., 2012, FPGA based microstepping scheme for stepper motor in space-based solar power systems, Industrial and Information Systems, IEEE Conference, 1-5

2. Bodson M., Sato J.S., Silver S.R., 2006, Spontaneous speed reversals in stepper motors, IEEE Transactions on Control Systems Technology, 14, 2, 369-373

3. Chen J.P., Cheng W., Han W., 2014, Analysis and simulation of stepper motor disturbance considering structural coupling, Applied Mechanics and Materials, 526, 103-108

4. Chen Y.M., 2010, Application of shape memory alloys to control the vibration of solar panels, Mechanical Engineering and Automation, 1, 173-175

5. Cur G.G., 2006, The satellite modeling and analysis of dynamic characteristic of drive system of solar array, Dissertation for the Master Degree in Engineering of Harbin Institute of Technology

6. Elsodany N.M., Rezeka S.F., Maharem N.A., 2011, Adaptive PID control of a stepper motor driving a flexible rotor, Alexandria Engineering Journal, 50, 2, 127-136

7. Kapun A., Hace A., Jezernik K., 2007, Identification of stepping motor parameters, International Conference on "Computer as a Tool", IEEE Conference, 1856-1863

8. Letchmanan R., Economou J.T., Tsourdos A., 2005, Modelling and analysis of geared PM stepping motor for simulation of all-electric off-road vehicles, Vehicle Power and Propulsion, IEEE Conference, 301-307

9. Luo Q., Li D., Zhou W., Jiang J., Yang G., Wei X., 2013, Dynamic modelling and observation of micro-vibrations generated by a Single Gimbal Control Moment Gyro, Journal of Sound and Vibration, 332, 19, 4496-4516

10. Si Z.H., LiU Y.W., Li K., 2010, Research on modeling and driver design of solar array drive assembly, Aerospace Control and Application, 36, 2, 13-19

11. Szolc T., Pochanke A., 2012, Dynamic investigations of electromechanical coupling effects in the mechanism driven by the stepping motor, Journal of Theoretical and Applied Mechanics, 50, $2,653-673$

12. XIA L., 1994, The primary research on low-frequency resonate of step-motor and its damping methods, Journal of Shenyang Polytechnic University, 16, 3, 73-78 
13. YAnG T.Y., WAng H., Xu F., 2007, Micro-stepping drive method study of two-phase step motors, Micromotors Servo Technique, 40, 9, 69-71

14. Yang Y.L., Cheng W., Wu S.J., Wang G.Y., 2010, Experiment and simulation of electromagnetic stiffness for stepper motor, Applied Mechanics and Materials, , 29, 1567-1573

15. Zhu S.Y., Lei Y.J., Wu X.F., Zhang D.P., 2015, Influence analysis on dynamic characteristic parameters of solar array drive system, Journal of Vibration Engineering, 28, 2, 183-189

16. Zhu S.Y., XIE Y., LeI Y.J., 2014, Characteristic analysis of disturbance aroused by solar array tracking drive, Journal of National University of Defense Technology, 36, 1, 27-33

Manuscript received July 16, 2015; accepted for print January 28, 2016 